\title{
Cryotherapy for low risk prostate cancer, oncological and functional medium term outcomes: A three center prospective study
}

\author{
Valerian Ciprian Lucan ${ }^{1^{*}}$, Franco Lugnani ${ }^{2 *}$, Salvatore Butticè ${ }^{3^{*}}$, Emre Sener ${ }^{4}$, Christopher Netsch ${ }^{5}$, \\ Michele Talso ${ }^{6}$, Francesco Cantiello ${ }^{7}$, Rosa Pappalardo ${ }^{3}$, Carlo Magno ${ }^{3}$ \\ ${ }^{1}$ Clinical Institute of Urology and Renal Transplants, Cluj-Napoca, Romania; \\ ${ }^{2}$ Unit of Urology, Kirurski Sanatorij Ljubljana, Slovenia; \\ ${ }^{3}$ Department of Human Pathology - Section of Urology, University of Messina, Italy; \\ ${ }^{4}$ Department of Urology, School of Medicine, Marmara University, Istanbul, Turkey; \\ ${ }^{5}$ Department of Urology, Asklepios Hospital Barmbek, Hamburg, Germany; \\ ${ }^{6}$ Department of Urology, Fondazione IRCCS Ca' Granda Ospedale Maggiore Policlinico, Università degli Studi di Milano, Italy. \\ ${ }^{7}$ Department of Urology, Magna Graecia University, Catanzaro, Italy. \\ * The Authors contributed equally to the manuscript.
}

\begin{abstract}
Summary Objectives: Analyze the oncologic and functional outcomes in patients affected by low risk prostate cancer underwent prostate cryotherapy. Materiasl and methods: It's a prospective tricentric study of 434 patients treated with prostate cryoablation for low risk prostate cancer. By low risk we refer to the D'Amico's risk classification. Two cycles of freezing/thawing are run for each patient following the technique described by Onik.

Results: For the 434 patients, the median age was 66 years with a standard deviation of \pm 6.68 , the average PSA was 6.17 $n g / d / L$, the median 5.55 with a standard deviation of \pm 2.13 , the mean prostate volume was $35.59 \mathrm{cc}$, the median $34.00 \mathrm{cc}$, with a standard deviation of \pm 7.89 . Biochemical failure occurred in 67 patients (15.4\%). Pre-operative erectile function in men was distributed as follows: severe in 95 patients (19.2\%), moderate in 95 (19.2\%), medium-moderate in 180 (36.4\%), mild in 92 (18.6\%), with no dysfunction in 32 (6.5\%) patients. Post-operative erectile function, measured 1 month after cryotherapy, was distributed as follows: severe in 321 (65\%) patients, moderate in 69 (14\%), medium-moderate in 79 (16\%), mild in 23 (4.7\%), and no dysfunction in only 2 patients (0.4\%). Post-operative erectile function after 3 months was distributed as follows: severe in 233 (47.2\%) patients, moderate in $66(13.4 \%)$, medium-moderate in $122(24.7 \%)$, mild in 65 (13.2\%), and no dysfunction in 8 patients (1.6\%). Urinary incontinence was present in 21 patients (4.8\%) after 3 months while it dropped to 13 patients (2.9\%) after 6 months. Conclusions: Cryotherapy in the treatment of prostate cancer remains a viable alternative. The availability of new cryoprobes and the use of new diagnostic means such as fusion magnetic resonance will make this more precise and more effective method.
\end{abstract}

KEY WORDS: Prostate cryotherapy; Minimally invasive treatment; Focal therapy; Low risk prostate cancer.

Submitted 2 January 2017; Accepted 18 February 2017

\section{INTRODUCTION}

In the era of the PSA screening the detected rate of prostate cancer has dramatically increased (1).
Currently there are many different options for treatments of prostate cancer, particularly for the low risk variety from D'Amico's risk classification (2).

According to the latest EAU guidelines there are different types of standard treatments for low risk prostate cancer. In recent years, with the aim of reducing the risk of overtreatment in this subgroup of patients, two conservative management strategies have been proposed: watchful waiting and active surveillance (3).

Surgical treatment of PCa consists of the radical prostatectomy (RP). In low risk patients the gold standard of care is the nerve sparing technique. The goal of surgery is to eradicate the disease by preserving continence and sexual potency when possible (4). Pelvic lymph node dissection (LND) is not required for low-risk tumors because the risk of positive lymph nodes does not exceed 5\%, according to Briganti's nomograms (5). Radiotherapy for prostate cancer is an important and safe alternative to surgery and is the only form of curative treatment (6-7). For the treatment of clinically localized prostate cancer new therapeutic approaches have emerged as alternative therapeutic options: defined focal therapies that include two methods, the HIFU (high-intensity focused ultrasound) and Cryotherapy (CSAP) (8-9).

The use of cryosurgery has been enhanced thanks to the introduction of a modern percutaneous approach using trans-rectal ultrasound probe introduced by Onik et al. in 1993 (8). In 2008 the AUA's best practice statement on cryosurgery affirmed that cryosurgery is an option for patients with an organ confined disease (10).

The aim of this study is to investigate the oncological and functional outcomes in patients affected by low-risk prostate cancer.

\section{MATERIALS AND MethodS}

Our is a prospective tricentric study of 434 patients treated with prostate cryoablation for low risk prostate cancer. By low risk we refer to the D'Amico risk classifi- 
cation of clinical stage $(<\mathrm{T} 2 \mathrm{a}$, PSA levels $<10.0 \mathrm{ng} / \mathrm{mL}$, and a Gleason score $\leq 6)$. All patients were operated on using the same surgical technique, performed by the same surgeon in $80 \%$ of cases. The surgery was performed under general anesthesia.

Patient preparation includes cleansing with enema and broad spectrum antibiotic prophylaxis. The patient is in the dorsal lithotomy position, in this way facilitating the exposure of the perineum and the movements of the rectal probe (Probe longitudinal biplane to $7.5 \mathrm{~Hz}$ ). In all cases the coaxial system Stryker Cryo/44 was used with cryoprobes of $2.4 \mathrm{~mm}$ in diameter, from six to eight in number depending on prostate volume. Cryoablation involves 2 cycles, one freezing and one cooling; respectively exploiting cycles of pressurized gas, argon (300 bar of pressure and $-180^{\circ} \mathrm{C}$ ) for the freezing cycle and helium for the heating cycle (200 bar of pressure with $180^{\circ} \mathrm{C}$ temperature exchange to $40^{\circ} \mathrm{C}$ in 30 seconds).

The temperature is monitored inside and outside of the prostate. Thermal sensors, are positioned in the apex, external sphincter, and neurovascolar bundle to the right and to the left of the gland.

Hydrodistention of the rectal area prostate was performed by injecting saline solution mixed with broad-spectrum antibiotic in the Denonvilliers' fascia (Onik maneuver band). Control cystoscopy is performed in order to ensure integrity of the urethra, which is protected by means of an FDA-approved continuous flow system with a pump pressure of 4.5 bar, which puts blue methylene physiological solution into circulation at $41^{\circ} \mathrm{C}$ and keeps adjacent tissues at a temperature of $38^{\circ} \mathrm{C}$ (11-14).

At each cryoablation two complete cycles of freezing/ thawing are run. Depending on prostate volume or on prostates with a larger longitudinal diameter of $35 \mathrm{~mm}$, a third cycle with a distal displacement of 10 millimeters of cryoprobes was necessary, in a maneuver referred to as "pull back". The patients were discharged within 24 hours, with a catheter in place for two weeks and were given antiinflammatory drugs for the pain (8).

Biochemical recurrence was determined according to the Phoenix defined by ASTRO criteria as a rising PSA, above the Nadir of more than $2 \mathrm{ng} / \mathrm{mL}$ (15).

Follow-up was carried out in hospital laboratories by monitoring PSA level every month for the first three months and then once every six months for a total of 54 months.

\section{Statistical analysis}

Statistical analysis was performed with Stata 14 software by StataCorp, descriptive variables were analyzed using mean, median, standard deviation, and a 95\% confidence interval (95\% CI). The survival curves were described using the Kaplan-Meier method. After making the appropriate test for normality (Shapiro-Wilk test) to validate if the assumptions underlying the model were statistically satisfied. We applied the model of analysis of variance in our sample.

\section{RESULTS}

For the 434 patients, the median age was 66 years with a standard deviation of \pm 6.68 , the average PSA was 6.17
Figures 1-2.

Box plot PSA/time.
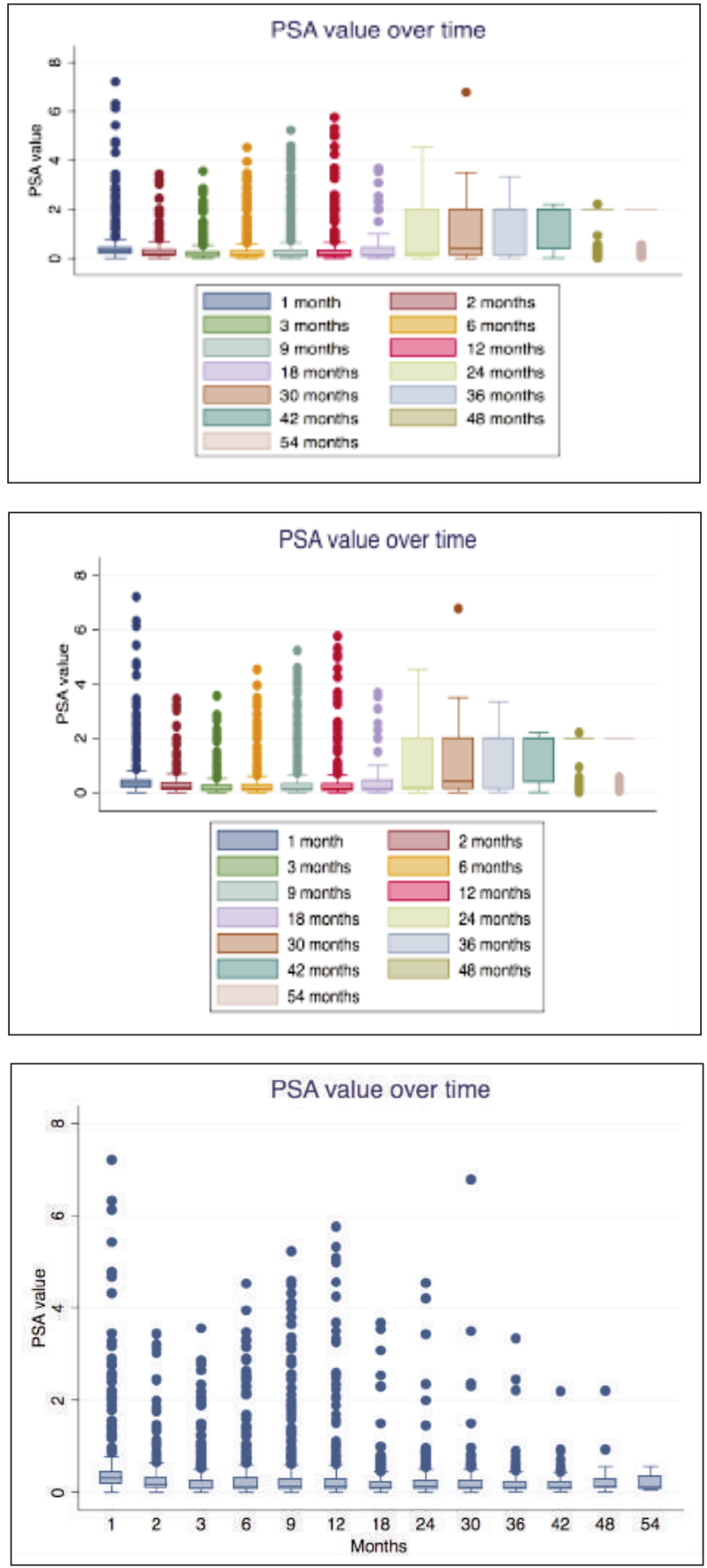

ng/dL, the median 5.55 with a standard deviation of \pm 2.13 , the mean prostate volume was $35.59 \mathrm{cc}$, the median $34.00 \mathrm{cc}$, with a standard deviation of \pm 7.89 .

Biopsies performed on patients for pre-intervention of disease diagnosis were conducted trans-rectally in 421 patients (97\%), and trans-perineally in 13 patients (3\%). Biochemical failure occurred in 67 patients (15.4\%) (Figures 1-2).

The survival curve it's shown in the Kaplan-Meier graph (Figure 3).

The average hospital stay was for 1.89 days, with a median of 1 and a standard deviation of \pm 1.48 . 
Figure 3.

Kaplan-Meier curve.

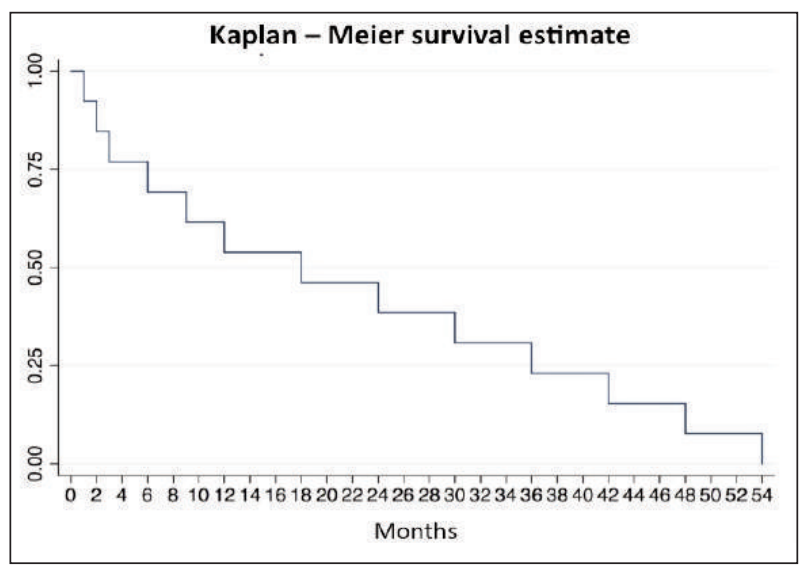

Pre-operative erectile function in men (Figure 4) was distributed as follows: severe in 95 patients (19.2\%), moderate in 95 (19.2\%), medium-moderate in 180 (36.4\%), mild in 92 (18.6\%), with no dysfunction in $32(6.5 \%)$ patients.

Post-operative erectile function, measured 1 month after cryotherapy (Figure 4), was distributed as follows: severe in $321(65 \%)$ patients, moderate in 69 (14\%), mediummoderate in 79 (16\%), mild in $23(4.7 \%)$, and no dysfunction in only 2 patients $(0.4 \%)$.

Post-operative erectile function (Figure 4) after 3 months was distributed as follows: severe in 233 (47.2\%) patients, moderate in $66(13.4 \%)$, medium-moderate in 122 $(24.7 \%)$, mild in $65(13.2 \%)$, and no dysfunction in 8 patients (1.6\%). In $87 \%$ of cases (377 patients) two freeze and thaw cycles were applied while three cycles were applied in the remaining $13 \%$ of patients (57 patients) due to the size and/or morphology of the prostate.

Number 4 needles were used in 68 patients (15.6\%), number 6 needles in 290 patients (67.4\%), and number 8 needles were used in 73 patients (17\%).

Urinary incontinence was present in 21 patients $(4.8 \%)$ after 3 months while it dropped to 13 patients (2.9\%) after 6 months.

\section{Figure 4.}

Diagram IIEF-5 Pre operative; Post operative; After 3 months.

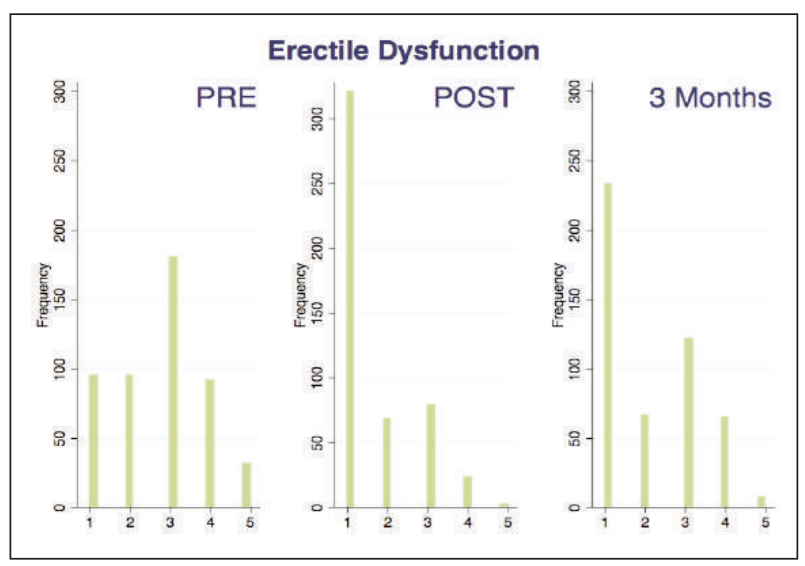

Concerning complications:

- Perineal hematoma in 207 (47.6\%),

- LUTS in 149 patients (34.3\%),

- Scrotal hematoma in 119 patients (27.4\%),

- Urinary tract infection in 44 patients (10\%),

- Perineal pain in 38 patients $(8.7 \%)$,

- Scrotal edema in 33 patients (7.6\%),

- Persistent hematuria was present in 22 patients (5\%),

- Urinary fistula in 16 patients (3.6\%),

- Urethral stricture in 14 patients (3.2\%),

- Hydronephrosis in only 1 patient $(0.2 \%)$.

\section{Discussion}

Cryotherapy for the treatment of prostate cancer is a technique that was originally introduced in 1960, but was soon abandoned because of excessive morbidity (11-13). The use of this therapeutic approach has increased with its reintroduction by Onik et al. in 1993 thanks to the introduction of a modern percutaneous approach with the aid of a trans-rectal ultrasound probe (8).

The treatment itself has undergone an evolution over decades of application in clinical practice; the development of interventional radiology has improved cryogenic technology and provided a better understanding of cryobiology (16-17).

The action mechanism of cryotherapy is complex. It exerts its effect by: the induction of protein denaturation, dehydration, rupture of the cell membrane due to expansion of ice crystals, the transfer of water from the intracellular side to extracellular spaces, vascular stasis, the induction of apoptosis, increasing the concentration of toxic substances at the intracellular level and finally osmotic shock (8, 11-14).

Traditionally, supporters of cryotherapy as primary treatment for prostate cancer have sustained that the procedure offers advantages over conventional treatments, allowing a non-invasive treatment of cancer in patients who are very elderly or are suffering from multi-comorbidity and would otherwise not be eligible candidates for traditional surgery; in recent years refinement of the procedure has also allowed an increase in effectiveness and safety.

Our data on survival free from biochemical recurrence after a follow-up of 54 months proves that this procedure produces efficacy rates that are highly competitive with all other conventional forms of therapy recommended by European and international guidelines; above all with three-dimensional radiation therapy, conformational radiotherapy or brachytherapy.

Our findings strongly indicate a need to expand the role of cryoablation in clinical practice of prostate cancer treatment. Head to head comparison with different modalities of treatment for prostate cancer is affected by several factors such as:

- the retrospective nature of the study,

- the monocentric nature of most studies,

- the often non-uniform or standardized selection of patients,

- the use of various dosing procedures and different techniques conducted by different laboratories,

- variations, according to different classification criteria, in the definition of biochemical failure. 
As a result, there is no definitive oncological comparative data. However, in a recent comparative study between cryoablation and radiation therapy conducted on 244 patients no significant differences between the two groups (76\%) were found (15).

In another trial a PSA nadir of less than $0.4 \mathrm{ng} / \mathrm{ml}$ in $81 \%$ of patients was shown without biochemical progression for 12 months of study.

In another single-center study with 590 patients and a mean follow-up of 7 years, the percentage of patients free of biochemical recurrence was approximately $61 \%$ (16). However, not all data in the literature support the idea that cryotherapy and radiotherapy are equivalent, especially in high-risk patients.

UAE guidelines, compared with AUA guidelines, show a substantial difference in classifying candidate patients for cryotherapy; in fact, in Europe, the "model" patient remains the low risk patient, whereas in the United States the procedure has been extended to cases of higher risk, as long as the disease is organ-confined.

Another aspect to consider is the comparison with invasive treatments such as radical prostatectomy. Gould et al. have shown a lower incidence of biochemical recurrence in patients who underwent cryotherapy compared to radical prostatectomy. This study presents several biases and was conducted on a small number of patients (18). Active surveillance is one of the treatment options for patients at low risk of prostate cancer.

Klotz et al. have analyzed patients ranging from $\mathrm{Tlc}$ and T2a, PSA $<10 \mathrm{ng} / \mathrm{mL}$, Gleason score $<6$, or PSA $<15$ $\mathrm{ng} / \mathrm{mL}$ for patients older than 70 years with Gleason $<7$ $(3+4)(19-20)$

With an average follow-up of 6.8 years, survival at 10 years was $97.2 \%$. For the $62 \%$ of patients who were still in active surveillance, $30 \%$ underwent radical prostatectomy; $10 \%$ preferred to switch to active treatment due to a PSA doubling time $<3$ years or an advancing Gleason score. The collective survival varies from $70 \%$ to $100 \%$. Biochemical failure has occurred in 13\% of patients undergoing active surveillance (21).

More recently a number of radical prostatectomies for low and intermediate risk patients demonstrated a percentage of PSA free survival from $60 \%$ to $65 \%$ and a cancer specific survival between $94 \%$ and $97 \%$ with a follow-up of 53 to 153 months. For high risk patients biochemical failure was $44 \%$ and $53 \%$ after 5 and 10 years respectively (22).

In 2010 Donnelly et al. published a randomized article that compared patients with localized prostate cancer treated with ERBT versus cryotherapy. They demonstrated no significant difference between the two techniques after 36 months in 244 patients with a mean follow-up of 100 months. The was some progression of the disease at 36 months in $23.9 \%$ of patients undergoing cryotherapy and $23.7 \%$ in patients receiving radiotherapy.

No difference was observed in the specific servival of the disease.

In another article, Donnelly et al. have also compared radical surgery, ERBT and brachytherapy with the data of cryotherapy in medium- and high-risk patients. The interval free of biochemical recurrence for medium risk patients was $37-97 \%$ for the radical prostatectomy, 26-
$60 \%$ for the ERBT and $66-82 \%$ for the brachytherapy. In high-risk patients the interval free of biochemical recurrence decreases to $16-61 \%$ in low-risk, $19-25 \%$ in the ERBT, and 40-65\% in brachytherapy (23).

With this data the authors concluded that cryotherapy seems superior to EBRT for moderate and high risk patients, and the data seemed comparable for mediumhigh risk patients undergoing brachytherapy and radical prostatectomy (15). In our series biochemical recurrence occurred in $15.4 \%$, with an incidence lower than many previously published trials.

In 2008, Cohen et al. reported 370 patients with a mean follow-up of 147 months and an interval free ofbiochemical recurrence in $80 \%, 74 \%$ and $46 \%$ respectively, for low, intermediate and high risk patients (24).

In our study we assessed erectile function, giving the patient the IIEF-5 test, we then evaluated the differences between the various categories. From the graphs you can see how classes with greater "movement" of patients towards worse erectile function in absolute terms are the patients with average to moderate dysfunction and slight during the pre-operative assessment phase, passing to the postoperative, respectively from 180 to 79 and 92 to 32 , while patients with severe dysfunction increased from 95 to 321 or from $19.2 \%$ to $65 \%$.

Radiation therapy appears to have a lesser impact on erectile function (25).

The percentage of maintained erectile function after various interventions was 0.76 after brachytherapy, 0.60 after brachytherapy + external beam irradiation, 0.55 after external irradiation only, 0.34 after nerve-sparing radical prostatectomy and 0.25 after standard radical prostatectomy. Selecting studies with a 2 year disease follow-up (excluding brachytherapy), the percentage was $0.60,0.52,0.25$ and 0.25 respectively (26).

In terms of quality of life, there are several studies that compare cryotherapy as a treatment for a locally advanced tumor. Patients treated with cryotherapy and brachytherapy reported a higher score of urinary disorders when compared with radical prostatectomy (27).

Since it was adopted, robotic prostatectomy has not shown significant benefits in functional outcome compared with the open approach. Ball et al. compared 719 patients treated with open, laparoscopic and a robotic approach, as well as brachytherapy and cryotherapy (28). Men treated with brachytherapy and cryotherapy were older and had more co-morbidities. The analysis demonstrated that cryotherapy has a negative impact on erectile function with respect to brachytherapy, and that this effect is reduced to 3 and 6 months, while the irritative and obstructive symptoms were higher in brachytherapy. In patients undergoing cryotherapy, worse sexual outcomes were demonstrated than in other treatments, but the baseline was also lower.

\section{Conclusions}

Cryotherapy in the treatment of PCa remains a viable alternative to more invasive approaches. The availability of new cryoprobes and the use of new diagnostic means such as fusion magnetic resonance will make this more precise and more effective method. 


\section{REFERENCES}

1. Neppl-Huber C, Zappa M, Coebergh JW, et al. Changes in incidence, survival and mortality of prostate cancer in Europe and the United States in the PSA era: additional diagnoses and avoided deaths. Ann Oncol. 2012; 23:1325-1334.

2. Jemal A, Siegel R, Ward E, et al. Câncer statistics, 2006. CA Cancer J Clin. 2006; 56:106-130.

3. Welty CJ, Cooperberg MR, Carroll PR. Meaningful end points and outcomes in men on active surveillance for early-stage prostate cancer. Curr Opin Urol. 2014; 24:288-92.

4. Ramsay C, Pickard R, Robertson C, et al. Systematic review and economic modelling of the relative clinical benefit and cost-effectiveness of laparoscopic surgery and robotic surgery for removal of the prostate in men with localised prostate cancer. Health Technol Assess. 2012; 16:1-313

5. Briganti A, Larcher A, Abdollah F, et al. Updated nomogram predicting lymph node invasion in patients with prostate cancer undergoing extended pelvic lymph node dissection: the essential importance of percentage of positive cores. Eur Urol. 2012; 61:480-7.

6. Kuban DA, Levy LB, Cheung MR, et al. Long-term failure patterns and survival in a randomized dose-escalation trial for prostate cancer. Who dies of disease? Int J Radiat Oncol Biol Phys. 2011; 79:1310-7.

7. Zelefsky MJ, Chan H, Hunt M, et al. Long-term outcome of high dose intensity modulated radiation therapy for patients with clinically localized prostate cancer. J Urol 2006; 176:1415-9

8. Onik G, Narayan P, Vaughan D, et al. Focal 'nerve-sparing' cryosurgery for treatment of primary prostate cancer: a new approach to preserving potency. Urology. 2002; 60:109-14.

9. Madersbacher S, Marberger M. High-energy shockwaves and extracorporeal high-intensity focused ultrasound. J Endourol. 2003; 17:667-72.

10. Babaian RJ, Donnelly B, Bahn D, et al. Best practice statement on cryosurgery for the treatment of localized prostate cancer. J Urol. 2008; 180:1993-2004.

11. Rees J, Patel B, Macdonagh R, et al. Cryosurgery for prostate cancer. BJU Int. 2004; 93:710-14.

12. Han KR, Belldegrun AS. Third-generation cryosurgery for primary and recurrent prostate cancer. BJU Int. 2004; 93:14-18.

13. Beerlage HP, Thüroff S, Madersbacher S, et al. Current status of minimally invasive treatment options for localized prostate carcinoma. Eur Urol. 2000; 37:2-13.

14. Long JP, Bahn D, Lee F, et al. Five-year retrospective, multiinstitutional pooled analysis of cancer-related outcomes after cryosurgical ablation of the prostate. Urology. 2001; 57:518-23.

15. Blana A, Brown SC, Chaussy C, et al. High-intensity focused ultrasound for prostate cancer: comparative definitions of biochemical failure. BJU Int. 2009; 104:1058-62.

16. Bahn DK, Lee F, Silverman $P$, et al. Salvage cryosurgery for recurrent prostate cancer after radiation therapy: a seven-year follow-up. Clin Prostate Cancer. 2003; 2:111-4.

17. Larson TR, Robertson DW, Corica A, et al. In vivo in- terstitial temperature mapping of the human prostate during cryosurgery with correlation to histopathologic outcomes. Urology. 2000; 55:547-552.

18. Gould RS. Total cryosurgery of the prostate versus standard cryosurgery versus radical prostatectomy: comparison of early results and the role of transurethral resection in cryosurgery, J Urol. 1999; 162:1653-1657.
19. Klotz L. Active surveillance for favorable-risk prostate cancer: who, how and why? Nat Clin Pract Oncol. 2007; 4:692-8.

20. Klotz L. Active surveillance for prostate cancer: trials and tribulations. World J Urol. 2008; 26:437-42.

21. Dall'Era MA, Albertsen PC, Bangma C, et al. Active surveillance for prostate cancer: a systematic review of the literature. Eur Urol. 2012; 62:976-83.

22. Yossepowitch O, Eggener SE, Bianco FJ Jr, et al. Radical prostatectomy for clinically localized, high risk prostate cancer: critical analysis of risk assessment methods. J Urol. 2007; 178:493-9.

23. Donnelly BJ, Saliken JC, Brasher PMA, et al. A randomized trial of external beam radiotherapy versus cryoablation in patients with localized prostate cancer. Cancer. 2010; 116:323-30.

24. Cohen JK, Miller RJ Jr, Ahmed S, et al. Ten-year biochemical disease control for patients with prostatecancer treated with cryosurgery as primary therapy. Urology. 2008; 71:515-8.

25. Fowler FJ, Barry MJ, Lu-Yao G, et al. Outcomes of externalbeam radiation therapy for prostate cancer: a study of Medicare beneficiaries in three surveillance,epidemiology, and end results areas. J Clin Oncol. 1996; 14: 2258-65.

26. Robinson W, Moritz S, Fung T. Meta-analysis of rates of erectile function after treatment of localized prostatecarcinoma. Int $J$ radiat Oncol Biol Phys. 2002; 54:1063-8.

27. Malcolm JB, Fabrizio MD, Barone BB, et al. Quality of life after open or robotic prostatectomy, cryoablation or brachytherapy for localized prostate cancer. International Braz J Urol. 2011; 37:118.

28. Ball J, Gambill B, Fabrizio MD, et al. Prospective longitudinal comparative study of early health-related quality of life outcomes in patients undergoing surgical treatment for localized prostate cancer: a short-term evaluation of fiveapproaches from a single institution. Journal of Endourology. 2006; 20:723-731.

\section{Correspondence}

Valerian Ciprian Lucan, MD

Lucan_valerian@yahoo.com

Clinical Institute of Urology and Renal Transplants, Cluj-Napoca, Romania

Franco Lugnani, MD

franco.lugnani@gmail.com

Unit of Urology, Kirurski Sanatorij Ljubljana, Slovenia

Salvatore Butticè, MD (Corresponding Author)

salvobu@gmail.com

Rosa Pappalardo, MD

gattoparto@hotmail.it

Carlo Magno, MD

cmagno@unime.it

Department of Human Pathology - Section of Urology, University of Messina Via Consolare Valeria 1, 98125, Messina, Italy

Emre Sener, MD

dr.emresener@gmail.com

Department of Urology, School of Medicine, Marmara University, Istanbul, Turkey

Christopher Netsch, MD

Department of Urology, Asklepios Hospital Barmbek, Hamburg, Germany c.netsch@asklepios.com

Michele Talso, MD

michele.talso@gmail.com

Department of Urology, Fondazione IRCCS Ca' Granda Ospedale Maggiore

Policlinico, Università degli Studi di Milano, Milan, Italy

Francesco Cantiello, MD

cantiello@unicz.it

Department of Urology, Magna Graecia University, Catanzaro, Italy 